\title{
Barry William Serpell
}

The month of death in this Obituary (BMJ 2016;355:i5613, doi:10.1136/bmj.i5613) is incorrect. It should be October. We apologise for this error. 